\title{
Ein Beitrag zur Analyse der Azinfarbstoffe
}

\section{Doctoral Thesis}

Author(s):

Bass, Rudolf

Publication date:

1932

Permanent link:

https://doi.org/10.3929/ethz-a-000090367

Rights / license:

In Copyright - Non-Commercial Use Permitted 


\title{
Ein Beitrag zur Analyse der Azinfarbstoffe
}

Von der
Eidgenössischen Technischen Hochschule in Zürich

zur Erlangung der

Würde eines Doktors der technischen Wissenschaften

genehmigte

Nr. 710

\author{
Promotionsarbeit
}

vorgelegt von

RUDOLF BASS

Dipl. Ingenieur-Chemiker

aus Celerina und Fuldera (Graubünden)

Referent: Herr Prof. Dr. H. E. Fierz

Korreferent: Herr Prof. Dr. L. Ruzicka

ZÜRICH :: 1932.

Diss.-Druckerei A.-G. Gebr. Leemann \& Co.

Stockerstr. 64. 


\section{Zusammenfassung.}

Durch Kondensation von p-Anisidin, p-Phenetidin und $\mathrm{p}$ Phenylendiamin mit $\beta$-Naphthol erhält man die entsprechenden Phenyl- $\beta$-Naphthylamin-Derivate. Kondensiert man diese mit Nitrosodimethylanilin, so bilden sich PhenylnaphthophenazoniumFarbstoffe mit besetzter p-Stelle zum Azonium-Stickstoff. Sie färben tannierte Baumwolle in violetten, lichtunechten Tönen an.

Kondensationsversuche von $\beta$-Naphthol mit den Echtblau R. R.-, B. B.- und Echtviolett B.-Basen verliefen resultatlos.

Erwärmt man die alkoholische Lösung von Neutralblau (C) mit konzentrierter Natronlauge und Wasserstoffsuperoxyd von $4 \%$, so bildet sich Dimethylnaphthosafraninon.

Durch Einwirkung von konzentrierter Salzsäure im Druckrohr erhält man aus:

Neutralblau (C)

Wollechtblau B L (By) $\rightarrow$ Oxy-rosindon (Naphthosafranol)

Novazolsäureblau G L (Gy) $\rightarrow$ Oxy-naphthosafranol.

Durch längeres Erhitzen mittels $60-70 \%$ iger Schwefelsäure am Rückflußkühler bei $155^{\circ}$ erhält man aus:

Neutralblau (C)

Basler Blau R (D H)

Wollechtblau B L (By)

$\longrightarrow \quad$ keinen neuen Körper

$\rightarrow$ keinen neuen Körper

Novazolsäureblau GL(Gy) $\rightarrow$ wahrscheinlich das Methylderivat der

Indocyanin B F (A)

Diaethylnaphthosafraninon-Sulfosäure

$\longrightarrow$ einen dem Dimethylnaphthosafraninon sehr ähnlichen Körper.

Durch Reduktion mit Zinn und Salzsäure konnte nur beim Wollechtblau BL (By) ein Spaltstück isoliert werden (Anilin). Bei allen anderen Farbstoffen verliefen die Versuche resultatlos.

Genannte Spaltreaktionen, an Farbstoffen mit besetzter pStelle zum Azonium-Stickstoff durchgeführt, zeigten negative Resultate; es konnten keine einheitlichen Körper isoliert werden. 